\title{
Efficient Machine Learning Techniques to Diagnose and Predict Alzheimer's disease
}

\author{
Sai Sindhuri Nasina, A. Rama Mohan Reddy
}

\begin{abstract}
Recent research in computational engineering have evidenced the design and development numerous intelligent models to analyze medical data and derive inferences related to early diagnosis and prediction of disease severity. In this context, prediction and diagnosis of fatal neurodegenerative diseases that comes under the class of dementia from medical image data is considered as the challenging area of research for many researchers. Recently Alzheimer's disease is considered as major category of dementia that affects major population. Despite of the development of numerous machine learning models for early diagnosis of Alzheimer's disease, it is observed that there is a lot more scope of research. Addressing the same, this article presents a systematic literature review of machine learning techniques developed for early diagnosis of Alzheimer's disease. Furthermore this article includes major categories of machine learning algorithms that include artificial neural networks, Support vector machines and Deep learning based ensemble models that helps the budding researchers to explore the scope of research in predicting Alzheimer's disease. Implementation results depict the comparative analysis of state of art machine learning mechanisms.
\end{abstract}

Keywords: Alzheimer's disease, Dementia, Deep learning, Machine learning, Artificial neural networks, Ensemble methods.

\section{INTRODUCTION}

Dementia is a health condition which is linked with memory thinking, behavior and the ability perform daily activities. Alzheimer's disease (AD) comes into the category of dementia which is associated with conditions of thinking impairment and loss in the performance either in the middle age or old age in general. The conditions by which we can determine this problem is by the existence of neurotic plaques within the brain and damage of brain cells progressively. In these conditions signs of dementia are progressed slowly and start troubling the daily life with highest impact. Even though in Alzheimer's disease the effects are higher in elderliness but it is not considered to be an old age condition. In the primary stages patients has a very little loss of memory eventually it will disrupt harmony of daily life. Available treatments cannot hold Alzheimer's disease (AD) from enhancing but due to early days of treatment can prevent the severity in their elderliness followed with healthy old age life. It is stated that the patients count with AD will double in the coming two decades [1], it is estimated that 1 in 5 will be affected in 2050 [2].

Revised Manuscript Received on February 05, 2020

* Correspondence Author

Sai Sindhuri Nasina*, Ph.D., Computer Science and Engineering, Sri Venkateswara University College of Engineering, Tirupati.

A. Rama Mohan Reddy, Professor,Computer Science and Engineering,S V University College of Engineering, Tirupati, India.

(C) The Authors. Published by Blue Eyes Intelligence Engineering and Sciences Publication (BEIESP). This is an open access article under the CC BY-NC-ND license (http://creativecommons.org/licenses/by-nc-nd/4.0/)
Due to this treatment with precision is required in early stages of AD. Alzheimer's disease is the reason for the $70 \%$ of dementia, which is creating havoc in

the public health among developed and developing countries. Dementia which is due to Alzheimer's disease is medically distinguished based on cognitive and executive dysfunction, psychiatric symptoms and behavioral problems. It results in trouble in daily functions which lead to deaths. In the last few years detecting Alzheimer's disease has become possible but it is confirmed only after complete postmortem in relation to brain autopsy.

Methods to treat the Alzheimer's disease patients are available since the $20^{\text {th }}$ century end via authorized ideas for cholinesterase inhibitors which shows ranges of disease affected in Alzheimer's disease D. Regular and constant medical supervisions help in coping up with the dementia symptoms but it will not change disease direction. In this decade there has been an incredible progress observed among the Alzheimer's disease of biomarkers discovery. Calculating these biomarkers in the existence of amyloid $\beta$ eta $(\mathrm{A} \beta)$ plaques in brain or in the cerebrospinal fluid, which is a regular practice observed in research. Due to biomarkers discovery we could differentia between dementia and Alzheimer's disease types. By observing the high levels of Alzheimer's disease biomarkers in the non-availability of medical signs it is stated to recognize the potential Alzheimer's disease patients in the early stage. This is the only in treatment of Alzheimer's disease before medical intervention as the as the last trials where failed.

When the drugs used are not successful it will make a fall out with the disease's modification in the initial stages. Major change is observed when change in the course of Alzheimer's disease could be observed clearly. This transformation towards secondary prevention from the indications of diseases can be termed as getting in between the pre-symptomatic at risk individuals in stopping or enhancing the disease onset. Due to the enhancements in the technology we can reach out to the large number of Alzheimer's disease patients and treat them in their enhancing stages as a preventive act. It is important to have a clear knowledge on machine learning before using it for analysis of Alzheimer's disease prognosis. Machine learning is part of artificial intelligence which has many features under its belt. Based on the past it estimates the new events and divides based on the obtained result. It obtains exact results in machine learning deep and thorough understanding of the techniques used is essential. Obtaining the methods and implementing it has a greater chance of seeing positive results when executed accurately and noting out every detail observations. 
To consider, all the methods involved in machine learning are little bit different when related to the general procedures.

For example few are designed based on certain data or on applications which is irrelevant for the different type of data. Due to this reason it is essential to contemplate multiple methods on a training data. There are three types of learning algorithms which are part of machine learning techniques they are: 1 . Supervised

learning 2. Unsupervised learning [3] 3. Reinforcement learning. For the given data the method which tries to draw a output by learning is known as Supervised learning. Understanding from a unclassified and unlabeled data by an self developed learning is known as Unsupervised learning. Coincidentally all the algorithms used in Alzheimer's disease are of supervised learning.

It is evidenced in several computations based literature studies that applications of machine learning techniques have been demonstrating significant progress in diagnosing and predicting the symptoms of Alzheimer's in the past few years. Several research studies indicated that application of artificial neural network (ANN) and support vector machine (SVM) and deep learning have demonstrated are extensively used for diagnosing Alzheimer's disease. The major difference in the context of ANN and SVM is optimization level of the solution generated. In case of ANN the solution id locally optimal and in SVM it is globally optimal [4]. It is suggested in few research studies that integration of autonomous and intelligent agents along with neural networks will demonstrate significant impact in diagnosing medical images. In the context of handling larger datasets deep learning could enhance the performance of prediction as it incorporates feature extraction as it primary step. Few researchers also used ensemble methods to enhance the accuracy of prediction. Henceforth there is need of a systematic study of various research articles in the context of analyzing the progress of research in the application of machine and deep learning techniques in diagnosing Alzheimer's disease in early stages.

The rest of this article includes a pragmatic research methodology [5] in section 2 that is adopted to execute the process of the systematic literature review of various studies that are experimentally implemented in diagnosing the Alzheimer's disease. Section 3 includes the necessity of Alzheimer's disease prediction techniques and classification of distinct prediction mechanisms. Furthermore includes detailed review and systematic analysis of various tools and techniques in diagnosing the Alzheimer's disease. Section 4 details about the research gaps and open research issues that are remained unaddressed.

\section{RESEARCH METHODOLOGY}

Pragmatic research methodology adopted in this article includes the process of systematic review (SLR) that is identified from [6]. Selection of the highly related research articles from the existing scientific databases viz IEEE, Springer. Science Direct, ACM and most cited articles from Scopus database is considered as a critical aspect in process of implementing a literature study. In this scenario, systematic study helps us to identify the most relevant articles to be reviewed relative to the Alzheimer's disease prediction. This systematic study enables the researchers to determine, validate and amalgamate the experimental studies deduced by various researchers working on Alzheimer's disease prediction. The research protocol followed in the SLR process is detailed as follows:

- Designing a review protocol that depicts complete process involved in the SLR Process that explores various dimensions of research in diagnosing the symptoms and predicting Alzheimer's disease in early stages.

- Formulate Research Questionnaire based on the evidenced PICO strategy to sort out evidence based research articles.

- Selection of the most relevant research articles relative to the Alzheimer's disease prediction.

- Generate synthesis of various selected literature studies to answer the research questionnaire.

Identify the unaddressed research challenges, open issues and research gaps intend towards diagnosing the Alzheimer's disease in medical image data.

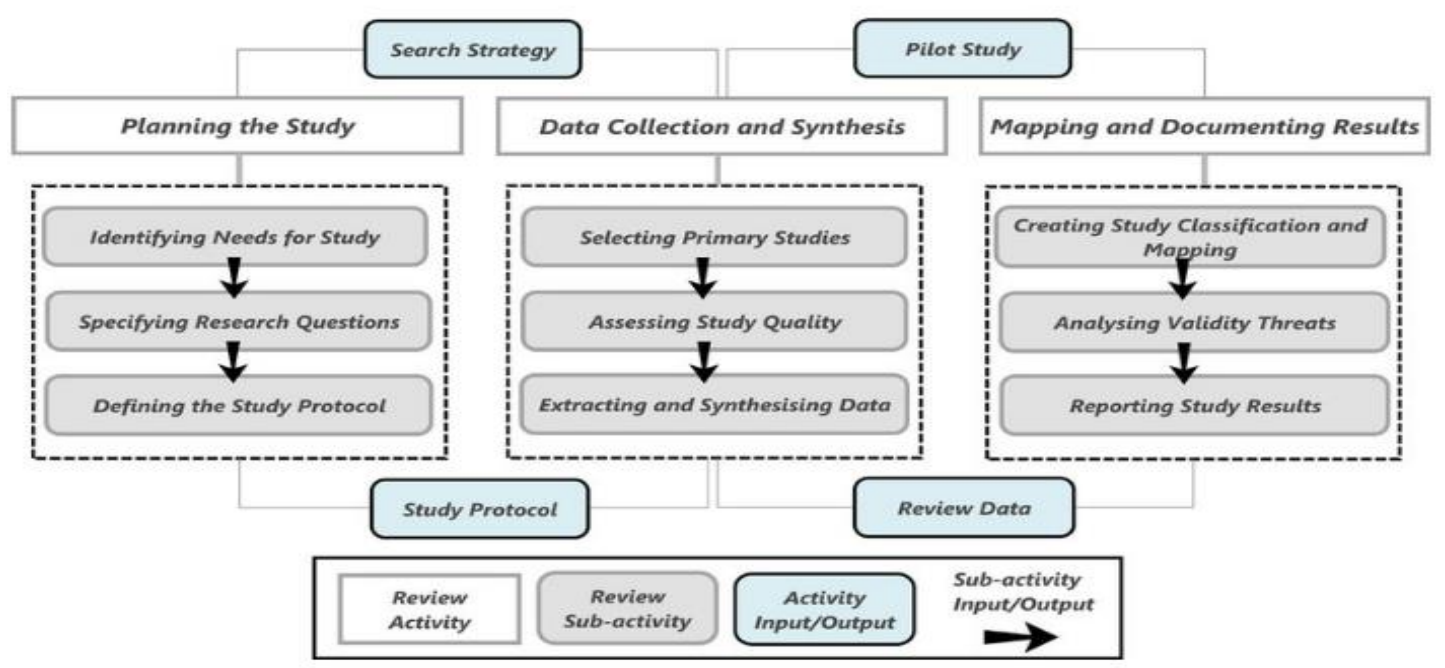

Figure 1: Research Methodology 


\begin{tabular}{|c|l|}
\hline $\mathbf{R Q}$ & $\begin{array}{l}\text { In what way Machine Learning and Deep learning } \\
\text { influences the accuracy of predicting Alzheimer's } \\
\text { disease in early stages }\end{array}$ \\
\hline $\mathbf{R Q}$ & $\begin{array}{l}\text { Enumerate various existing techniques that are } \\
\text { devised for accurate prediction of Alzheimer's } \\
\text { disease }\end{array}$ \\
\hline $\mathbf{R Q}$ & $\begin{array}{l}\text { What are various datasets and tools utilized for } \\
\text { experimental interpretation in existing research } \\
\text { studies }\end{array}$ \\
\hline $\mathbf{R Q}$ & $\begin{array}{l}\text { What is the current research progression and open } \\
\text { research challenges related to diagnosis of } \\
\text { Alzheimer's disease symptoms in premature stages }\end{array}$ \\
\hline
\end{tabular}

\section{A. Research Questions}

The most important phase in the SLR process is developing a research questions that primarily sets and objective for the literature review. Formulating absolute research question streamline the study towards relevant research. In this article, PICO strategy [7] is applied in generating research questionnaire that elevates the quality of the study. The research questionnaire generated on Alzheimer's disease predictions as follows:

\section{B. Search Strategy}

The main intention of this study is to identify, analyze and accumulate the relevant research works related to the prediction of Alzheimer's disease in the medical image data using the principles of evidence based research stated in [8]. In this process, data collection and extraction will be executed on the well known scientific databases and indexed journals. The combinations of the search strings are notated as shown in Table 1.

Table 1: Search String employed to conduct SLR process

Alzheimer's disease OR Dementia OR thinking impairment

AND

Prediction OR multi-objective analysis $\mathbf{O R}$ multivariate analysis OR Optimization Or Diagnosis

AND

Classification OR Clustering

AND

Artificial intelligence OR Artificial Neural networks OR

Time series prediction models

AND

Hybrid Models OR Deep learning Models OR Machine learning Models OR Fuzzy Models

AND

Systematic Study OR SLR OR Mapping Study OR Review

In the preliminary searching mechanism, on using above combination of search strings of the scientific databases that include Springer, IEEE, Science Direct, ACM and Scopus indexed with high citations around 172 research articles are identified in specific to the diagnosing the dementia and Alzheimer's disease symptoms in medical image data based on various technical indices. Table.2 enumerates the quantity of the research papers obtained from various scientific databases.

Table 2: No. of Research articles obtained from various scientific databases

\begin{tabular}{|c|c|}
\hline Database & No. of Papers \\
\hline IEEE & 34 \\
\hline ACM & 22 \\
\hline SPRINGER & 36 \\
\hline SCIENCE DIRECT & 35 \\
\hline GOOGLE SCHOLAR & 45 \\
\hline Total & $\mathbf{1 7 2}$ \\
\hline
\end{tabular}

\section{Selection of the relevant research articles}

In the previous phase of the data collection, around 172 relevant research articles are extracted from scientific databases based on the interpreted search strings. It is observed that, there is a need of a data filtering mechanism that scrutinizes the relevant research articles based on the relevancy rate of the title and abstract related to problem statement. Furthermore in the initial stage it-self the articles are to be scrutinized based on the inclusion and exclusion criteria specified in Table 3 . Finally 32 most relevant research articles are considered for conducting the systematic review.

Table 3: Inclusion and Exclusion Criteria

\begin{tabular}{|c|c|}
\hline Inclusion Criteria & Exclusion Criteria \\
\hline $\begin{array}{l}\text { Articles that include classification } \\
\text { and clustering mechanisms along } \\
\text { with ANN, AI, Fuzzy models and any } \\
\text { other hybrid prediction mechanisms }\end{array}$ & $\begin{array}{l}\text { Ambiguous articles } \\
\text { without specifying } \\
\text { implementation details } \\
\text { of the proposed } \\
\text { mechanisms and } \\
\text { articles those are } \\
\text { purely theoretic. }\end{array}$ \\
\hline $\begin{array}{l}\text { Evidence-based research articles with } \\
\text { detailed description of } \\
\text { implementation details along with its } \\
\text { tools, datasets and test beds }\end{array}$ & $\begin{array}{l}\text { Lecture notes, white } \\
\text { papers and theoretic } \\
\text { conference articles } \\
\text { related to the } \\
\text { Alzheimer's disease } \\
\text { prediction }\end{array}$ \\
\hline $\begin{array}{l}\text { Articles that are primarily } \\
\text { implemented in the electronics and } \\
\text { computer engineering domain }\end{array}$ & \multirow{2}{*}{$\begin{array}{c}\text { Articles are written in } \\
\text { other than the English } \\
\text { language }\end{array}$} \\
\hline $\begin{array}{l}\text { Articles that are written in the } \\
\text { English language }\end{array}$ & \\
\hline
\end{tabular}

\section{MACHINE LEARNING TECHNIQUE FOR DIAGNOSING ALZHEIMER'S DISEASE}

\section{A. SVM based Techniques:}

For classification and regression problems support vector machine is a notable method you find to use for its stability and wide usage. Good performance is achieved by SVM due to Structural Risk Minimization principle. It uses maximum margin principle to differentiate data points. Kernel functions are used to categorize non linear data to higher data points.

To increase the productivity numerous variants are introduced in relationship with generalization ability and training time. 
Few computational efficient variants are twin support vector machines and Least squares based twin support vector machine.

\section{a. Image modality using SVM:}

In distinguishing MRI images image modality is a very important one.T1 images are considered mostly for structural images when compared to T2 images. Delineation found in the ventricular surface of brain because of atrophy can be detected accurately in T1-weighted images. Due to this researchers prefer to T1 images.

\section{b. Feature selection and extraction with SVM}

Lover et al. [9] stated that positron emission tomography (dog) scans will provide added source to MRI scans in which classification precision of CN vs MCI making use of SVM happens to be increased . Dukart et al. [10] have advantages on this proven fact that fluorodeoxyglucose-PET (FDG-PET) properties is differentiable once associated with MRI. Further, optimism try higher in $\mathrm{CN}$ vs $\mathrm{AD}$ [11] with PET photographs (100 \%) whenever related to single photon emission determined with tomography (SPECT) photographs (97.5 \%). Coherent answers are found for CN vs AD [12] with more accurate for PET images (96.67 \%) when appropriate to images that are SPECT94.5 per cent).Kamathe et al. [13] merged T1, T2 and proton occurrence (PD) goes through into areas for CN vs post. Hojjati et al. [14] used state that is resting MRI (rs-fMRI) to obtain the connectivity changes in brain for classification of MCIc vs MCInc, while Sheng et al. [15] utilized connection info from fMRI information. a parcellation that is enhanced is presented in [16] for identifying lesser alterations in grey point (GM). Magnin et al. [17] put forward an attribute option way which utilizes histogram of welfare (ROIs) for CN vs advertising. Gerardin et al. [18] with services of form qualities regarding hippocampus to differentiate $\mathrm{CN}$, MCI and $\mathrm{AD}$, and found summation shape that is stating functions were considerable in comparison to volumetric services. Normalized suggest square error (NMSE) properties can be used [19] in distinguishing CN with very early offer. A clustering oriented approach illustrated [20] for a combined band of adjacent voxels in division of CN, MCI and offer. Fisher discriminate ratio (FDR) is considered [21] in identifying voxels that are useful properties (VAF) from SPECT imagery Gaussian blend unit (GMM) is utilized in [22] for CN vs AD. GMM dependent feature draws the actual data linearly separable was proposed. dog and sMRI information is regarded as by Ortiz et al. [23] in knowing the best distinguishing the items utilized considering simple inverse covariance estimation (SICE) way with SVM. In classification of $\mathrm{CN}$ vs post, Non-negative matrix factorization (NMF) are optimum in comparison to PCA eith SVM counting on their items.

Moreover, Abdulkadir et al. [24] described the total results of hardware heterogeneity on the category precision of SVM. They can find the confidence that is high of category abilities for large trials. SPM properties for CN, MCIc, MCInc, and advertising aren't competitive with DARTEL which can be recommended by Cuingnet et al. [25] significantly less classification reliability try obtained by function option tips when accomplished for sMRI photos for the reason that improvement of hyperparameters. Moreover levels situated features are thought to better than voxel based morphometry (VBM) as stated by Schmitter et al. [26].
Morphological features of brain parts are used by Plocharsky et al. [27] to branch <blank> CN vs advertising, while extended et al. considered shape distinctions as issues' of mind in categorizing of $\mathrm{CN}, \mathrm{AD}$, sMCI, and pMCI.

Many performs come from the Wavelet established qualities. Chaplot et al. [28] utilized wavelet that is discrete (DWT) characteristics, while Zhang et al. [29] inferred that 3-D DWT and SVM become useful in identifying of $\mathrm{CN}$, MCI and advertisement subjects. Segovia et al. [30] found least that is partial (PLS) equipment provides larger FDR rating whenever linked to principal component assessment (PCA) for CN vs AD using SPECT photos. Ortiz et al. utilizes self organizing maps (SOMs) [31] among unsupervised segmentation of sMRIimagesin distinguishing of CN vs post. However, Chaplot et al. [28] arrived to know that SVM performance is actually higher to SOM once identifying of Alzheimer's patients predicated on T2-weighted images. Techniques like SVM-RFE [32, 33] utilize feature that is optimized approach in [34] to select prominent brain characteristics for $\mathrm{CN}$ vs AD. Separate component research (ICA) employs works that are many, 36] for distinguishing of $\mathrm{CN}$ vs advertising using SVM. EEG information is furthermore ideal for distinction of $\mathrm{CN}$ vs advertising using SVM. Mazaheriet al. [37] used EEG tracks from phrase awareness oriented subject areas in category of MCIc from MCInc and CN.

\section{B. ANN based Mechanisms}

Artificial neural networks (ANN) presented considers machine learning models for highly nonlinear patterns of data. Here we reviews the methods which rely on ANNs and other methods.

\section{a. Transfer Learning}

Traditional machine training designs if the wide range of products offered is extremely lowest it considers samples of a unmarried domain name anywhere their performance is actually very impacted. The strategy in which types of target website and other reliable domains tend to be regarded is proven to be move reading. a manifold that is multimodal exchange studying (M2TL) is reported by Cheng et al. [38] with the purpose to convert understanding acquired from a reliable site (CN against $\mathrm{AD}$ ) to simply help in mastering the mark domain (MCIc vs MCInc). Further jobs of Cheng et al. [39] directly came with an idea of a book multi-domain transfer learning model (MDTL).

Deep transfer learning is used by Hon et al. [40] to carry out sample that is small difficulty in teaching deep neural systems by changing two common pre-trained networks artistic geometry cluster 16 (VGG16) and beginning from the target chore of classifying advertising and $\mathrm{CN}$ issues. TrAdaBoost formula is considered to transmit info TrAdaBoost formula by Zhou et al. [41]. Shifting insights without looking at problems leads to class and degradation labeling would be completely wrong. In passing these circumstances, Robust multi-label transfer learning (rMLTFL) approach which modifies the first labeling on to a multiple bit tag programming vector are suggested by Cheng et al. [41]. 
Li et al. [43] transfers' facts extracted from ADNI trials into the trials acquired locally through the subspace positioning algorithm.

\section{b. Feature Selection Method}

Most practices are reported for better feature selection (FS) from neuroimaging information. Ahmadlou et al. [44] suggested non-linear services which may reveal noteworthy distinctions among particular EEG sub-bands however may well not aid in categorizing the 2 teams (CN and advertisement) within band-limits of EEG. To take care of this, exposure graphs (VG) are considered in services extraction from EEG indicators. Short period of time Fourier change (STFT) and WT attributes include used Rodrigues et al. [45] to categorize according to ANN. a trait of AD is the reduction in GM and WM areas which leads in diminishment of varied parts and WM tracts loss of GM and WM volume. This is also followed closely by boost in size of ventricles and CSF levels. Yang et al. [46] created a category framework for which GM, WM are together. CSF volumetric properties can be found in ventricular $2 \mathrm{D}$ and shape that is 3D. Using surface formulated morphometry to get down features that are cortical choice method for removing properties from MR artwork. Making use of cortical depth data Cho et al. [47] stated robust classification method that is incremental. Using manifold transform that is harmonic is changed into spatial regularity. Various other types of information like neuro-psychological and physiological studies, hereditary makeup and class tends to be examined beside with MRI SCANS.

Using ANN Quintana et al. [48] improved data from neuropsychological reports, era and training in differentiating MCI and post. a book wrapper feature choices method was claimed by Chyzyk et al. [49] which gather GA along serious training machine (ELM). Into the works that are continuous et al. [50] regarded volumetric and shape services like a team. A novel method is stated to select important ROIs using SOM by Ortiz et al. [51] Wang et al. [52] used a hybrid PSO with the artificial bee colony (ABC) optimization algorithm along with a feed forward neural network (FFNN) in pointing out the problems of prior knowledge in manual ROI selection and high dimensionality in whole brain multivariate analysis. Removing properties from particular ROIs with the mind is just a method that is famous dealing the difficulty of large dimensionality of entire head research to extract services from certain ROIs on the brain. Element past knowledge will come as being a drawback. Table 4 shows the summary of big studies in earlier three years

Table 4 in Summary of Significant studies in past three years

\begin{tabular}{|c|c|c|c|c|c|c|c|c|c|c|}
\hline \multirow{2}{*}{ Year } & \multirow{2}{*}{ Authors } & \multirow{2}{*}{ Target } & \multirow{2}{*}{ Modality } & \multirow{2}{*}{$\begin{array}{c}\text { Feature } \\
\text { extraction }\end{array}$} & \multirow{2}{*}{$\begin{array}{l}\text { Machine } \\
\text { learning }\end{array}$} & \multirow{2}{*}{ Dataset } & \multirow{2}{*}{ Validation } & \multicolumn{3}{|c|}{ Performance } \\
\hline & & & & & & & & \begin{tabular}{|l|} 
Acc $(\%)$ \\
\end{tabular} & Sens (\%) & Spec (\%) \\
\hline 2016 & Moller et al. & CN vs AD & sMRI(T1) & VBM & SVM (Linear) & $178(94 \mathrm{CN}, 84 \mathrm{AD})$ & LOOCV & 85 & 83 & 87 \\
\hline 2016 & Plocharsky et al. & CN vs AD & sMRI (T1) & $\begin{array}{l}\text { Morpholgical } \\
\text { features (length, } \\
\text { area, depth) }\end{array}$ & SVM (Linear) & $210(100 \mathrm{CN}, 110 \mathrm{AD})$ & 10 -fold & 87.9 & 90 & 86.7 \\
\hline \multirow{3}{*}{2017} & \multirow{3}{*}{ Alam et al. } & CN vs $\mathrm{AD}$ & \multirow{3}{*}{$\mathrm{sMRI}(\mathrm{T} 1)$} & \multirow{3}{*}{ VolBM+KPCA } & \multirow{3}{*}{$\begin{array}{c}\text { SVM (Multiple } \\
\text { kernel) }\end{array}$} & \multirow{3}{*}{$\begin{array}{c}293(102 \mathrm{CN}, \\
102 \mathrm{MCI}, 89 \mathrm{AD})\end{array}$} & \multirow{3}{*}{10 -fold } & 93.85 & 92.1 & 94.45 \\
\hline & & CN vs $\mathrm{MCI}$ & & & & & & 86.54 & 84.85 & 87.74 \\
\hline & & MCI vs AD & & & & & & 75.12 & 73.92 & 77.24 \\
\hline \multirow[t]{2}{*}{2017} & \multirow[t]{2}{*}{ Khedher et al. } & CN vs MCI & \multirow[t]{2}{*}{$\mathrm{sMRI}(\mathrm{T} 1)$} & \multirow[t]{2}{*}{ ICA } & \multirow{2}{*}{$\begin{array}{l}\text { SVM } \\
\text { (RBF) }\end{array}$} & \multirow{2}{*}{$\begin{array}{c}818(229 \mathrm{CN}, \\
401 \mathrm{MCI}, 188 \mathrm{AD})\end{array}$} & \multirow[t]{2}{*}{$k$-fold } & 79 & 82 & 76 \\
\hline & & MCI vs $\mathrm{AD}$ & & & & & & 85 & 85 & 86 \\
\hline \multirow{2}{*}{2017} & \multirow{2}{*}{ Beheshti et al. } & CN vs $\mathrm{AD}$ & \multirow{2}{*}{ sMRI (T1) } & \multirow{2}{*}{$\mathrm{VBM}+\mathrm{GA}$} & \multirow{2}{*}{ SVM (Linear) } & \multirow{2}{*}{$\begin{array}{c}458(162 \mathrm{CN}, 65 \mathrm{sMCI} \\
71 \mathrm{pMCI}, 160 \mathrm{AD})\end{array}$} & \multirow{2}{*}{10 -fold } & 93.01 & 89.13 & 96.8 \\
\hline & & pMCI vs sMCI & & & & & & 75 & 76.92 & 73.23 \\
\hline \multirow{3}{*}{2017} & & CN vs AD & & & & & & 96.5 & 93.85 & 97.78 \\
\hline & Long et al. & CN vs pMCI & sMRI (T1) & $\mathrm{MDS}+\mathrm{PCA}$ & SVM (Linear) & 427 (135 CN, $132 \mathrm{sMCI}$ & 10 -fold & 97.1 & 87.37 & 94.82 \\
\hline & & sMCI vs pMCI & & & & $95 \mathrm{pMCI}, 65 \mathrm{AD})$ & & 88.99 & 86.32 & 90.91 \\
\hline 2017 & Tangaroetal & CNvs AD & sMRI(T1) & VolBM & SyM(Linear) & $372(117 \mathrm{CN}$ & 10-fold & 100 & - & - \\
\hline 2017 & Lu et al. & CN vs MCI & FDG-PET & VBM & RF-RSVM & $272(152 \mathrm{CN}, 120 \mathrm{MCI})$ & 3-fold & 90.53 & 90.63 & 93.33 \\
\hline 2017 & Alam et al. & CN vs $\mathrm{AD}$ & sMRI & DTCWT/LDA & TWSVM & $237(130 \mathrm{CN}, 137 \mathrm{AD})$ & 10 -fold & 96.88 & 97.72 & 95.61 \\
\hline 2017 & Hojjati et al. & MCIc-MCInc & rs-fMRI & PCC+F-score & SVM (Linear) & 80 (18 MCIc, 62 MCInc) & 9-fold & 91.4 & 83.24 & 90.1 \\
\hline 2017 & Kulkarni et al. & CN vs AD & EEG & $\begin{array}{l}\text { ICA/ Wavelet/ } \\
\text { Spectral }\end{array}$ & SVM & $100(50 \mathrm{CN}, 50 \mathrm{AD})$ & LOOCV & 96 & - & - \\
\hline & & CN vs AD & & & & & & 95.1 & 93.8 & 83.8 \\
\hline & Sunetal & CN vs MCI & & $\mathrm{VBM}+\mathrm{PCC}$ & & $509(162 \mathrm{CN}, 134 \mathrm{sMCI}$ & & 70.8 & 72.1 & 69.1 \\
\hline 2018 & Sun et al. & sMCI vs pMCI & sMRI(T1) & $\mathrm{VBM}+\mathrm{PCC}$ & SVM & $76 \mathrm{pMCI}, 137 \mathrm{AD})$ & 5-fold & 65.4 & 67.6 & 64.2 \\
\hline & & MCI vs AD & & & & & & 65.7 & 63.2 & 67.3 \\
\hline
\end{tabular}

\section{Deep Learning technique for predicting Alzheimer's disease}

Diagnosis accuracy of post, MCI and MCIc can be improved as put on SAE as previously mentioned by Suk et al. [53] it reveals correlations that are nonlinear. Suk et al. [54] used patch-basedcharacteristics of MRI, and PET in differentiation of advertisement and MCI from CN by strong Boltzmann maker (DBM). 3D-CNN performance is great in classification of AD, MCI from CN when SAE and CNN are positioned collectively through the learn of Payan et al. Suk et al. [56 ] made use of DL-based figures to describe advertisement, MCI, CN, and MCIc from MCInc having an classifier that is SAE-based. Obtaining advanced level properties and soft-max logistic regressor Liu et al. [55] utilized SAE based DL architecture to cure AD in four levels. to differentiate advertisement and MCI fromCN, (LR). Hosseini et al. [57] claimed ahighly checked adaptive 3D-CNN (DSA-3DCNN) which is trained before by 3D convolutional autoencoder (3D-CAE) centered on MRI.. Ortiz et al. [58] Work by Ortiz et al. [58] is that dependent on mechanized anatomical naming (AAL) chart book 3D

patches are gotten and prepared DBNs. 
At the point when highlights are separated utilizing DBN (FEDBN-SVM) engineering best characterization exactness is accomplished. Three biomarkers for Alzheimer's sickness determination for example X-ray, PET, CSF and so on are assembled to recognize solid and AD members by D. Zhang et al. (2011).Among 202 occasions, 51 were AD, 99 were $\mathrm{MCI}$ and 52 were controls that are solid is pattern information used by the creators. Numerous tests were held for MRI, PET and CSF and the blend of these utilizing 10 crease cross approval. $93.2 \%$ with $93 \%$ affectability is the precision for characterizations and $93.3 \%$ explicitness is because of the fellowship of these modalities when individual test has recorded most elevated exactness of $86.5 \%$. Predictable robustis and improvement noted by multimodal characterization technique (utilizing all MRI, PET, and CSF) in view of the creator over number of chosen mind areas. The informational collection of $96 \mathrm{AD}$ and 273 sound controls, marked by master doctors , FDG-PET can be costly and it is interesting to see how the system of Zhang et al. is applied with simply the two MRI and CSF, yet this data isn't displayed. Cuingnet R1, 2011 strategy has gotten most noteworthy when contrasted with the vaue of $91.8 \%$ consolidated to MRI and CSF. It is simpler to lead R. Chaves et al., (2010) to present a characterization strategy for starting finding than Single Modality Approach. The connections between properties of the pre-handled collections that are informational recovered by affiliation rule mining

\section{RESULTS AND DISCUSSION}

While in the representation research, each unit carried out 68 occasions in the simulation experiment according to different tests sets, whenever VBSD dataset can be used 36 occasions and Dem@Care is employed 32 circumstances. Each fresh outcome is determined in line with the integration of all of the testing arranged identification listings. Figure shows that the visualization of this experimental outcomes of various metrics and sizes. Through the fresh results, it may be viewed that the LogisticRegressionCV, LinearSVC and MLP versions perform well in both the VBSD dataset while the Dem@Care dataset. Through the experimental effects, LogisticRegressionCV gotten the finest performance weighed against more category practices. Included in this, F1-Score achieved 86.9\% throughout the VBSD dataset and 89.4\% from the Dem@Care dataset.

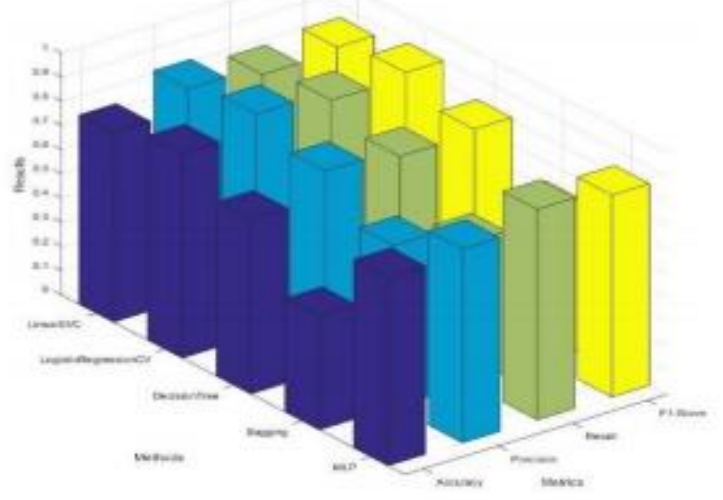

Figure 2: Simulation of Machine learning Models to predict Alzhemer disease

LinearSVC and MLP models play fairly really that their unique reliability attained above $70 \%$. Nevertheless,
DecisionTree and Bagging obtained poor overall performance because DecisionTree can be so easily producing a model that is complex that renders the generalization potential conduct not really. Additionally, moreover it comes with the disadvantage of uncertainty. Limited changes of data can lead to different tree generation. The training sets and testing sets in this simulation experiment are located in continual modification, and decisionTree classifier performs thus badly within this simulation experiment. The bottom classifier of Bagging picks DecisionTree classifier, therefore the results of Bagging generally is dependent on the reliability associated with base classifier. Given that benefit, the classification efficiency of Bagging can also be bad. The distinctions in code and enunciation between countries aren't regarded as in mixing the two datasets in simulation experiment.

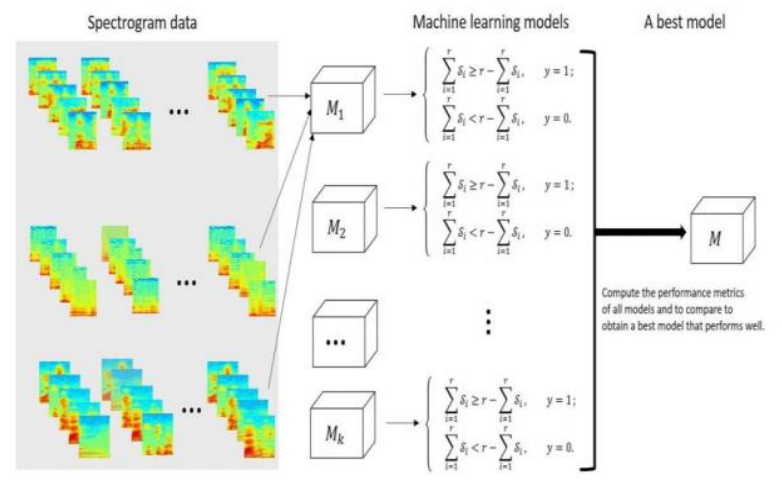

Figure 3: Deriving efficient machine learning model for predicting Alzheimer disease

\section{CHALLENGING ISSUES AND RESEARCH GAP}

The studies which are briefed in the last section are few examples stating the method to conduct machine learning experiments and the results are impressive. These studies claimed the procedure of validation on outcome and explained the prognosis and prediction of AD. Yet identifying the potential issues of input data for implementation and validation for various studies is essential. When the analysis of the described studies are reviewed the input size, attributes and validation are the most common problems by using small data sets higher accuracies can be obtained easily but those procedures are simple . Overtraining is prone with small data set and with large data size several results like robustness, accuracy and reproductivity is observed.

Research is principally on pathologically dubious information which can present equivocalness. The ascribes to example proportion have sway on results. In the past examinations, consideration is gesture paid on the number and general data of traits. Information quality and significant property determination are vital in AI for successful outcomes age. Sadly, the creators once in a while portrayed the strategies used to guarantee the information honesty and quality. Choice of highlight is significant for information quality. In any case, the highlights picked for some clinical information, for example histological evaluations may not be pertinent after some time.

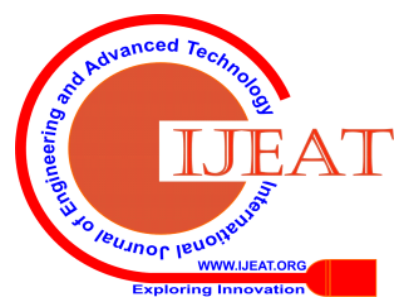




\section{CONCLUSION}

This article mainly focuses on presenting the detailed review of research works that includes various techniques and intelligent models developed for early diagnosis of Alzheimer's disease. In this study, research works based on major categories of machine learning techniques like artificial neural networks, Support vector machines and Deep learning based ensemble models are reviewed in detailed manner. Apart from that, techniques based on ensemble models and transfer learning is discussed. It is inferred from the systematic literature review that current trend of research in predicting Alzheimer's disease is focused on the feature extraction than the clustering and classification phase which can be considered a future scope of research for accurate diagnosing Alzheimer's disease.

\section{REFERENCES:}

1. D, Zhang, 2011. Multimodal plan of Alzheimer's condition and gentle subjective weakness. Neuroimage, 55(3), 856-867.

2. Brookmeyer, Ron, Elizabeth Johnson, Kathryn ZieglerGraham, and H. Michael Arrighi, 2007. Deciding the Internationally Stress of Alzheimer's Disease Illness. Alzheimer's disease and Dementia, 3.3, 186-191.

3. Duda RO, Hart PE, Stork DG. , 2001. Sample arrangement Alzheimer'(second variation). Nyc: Wiley.s and Dementia, 2nd type

4. T, Mitchell , 1997. AI. Nyc: McGraw Hill, 2nd adaptation, 135

5. Supekar, K, Menon V, Rubin D, Musen M, Greicius MD, 2008. System study of built-in functional cerebrum

6. network in Alzheimer's malady. . PLoS Comput Biol , 4(6), 1-11.

7. Cruz, J.A. what's more, D.S. Wishart, 2006. Has of maker Learning in Cancer forecast and Prognosis.Cancer Informatics, 2, 5977.

8. Ahmed Abdulkadir, Bénédicte Mortamet, Prashanthi Vemuri, Clifford roentgen Jack Jr, Gunnar Krueger, Stefan Klöppel, Alzheimer's ailments Neuroimaging Initiative, et al. 2011. Impacts of devices heterogeneity regarding the presentation of SVM Alzheimer's malady classifier. Neuroimage 58, 3 (2011), 785-79

9. B Al-Naami, N Gharaibeh, and An AlRazzaq Kheshman. 2013. Computerized recognition of Alzheimer issues making use of locale developing procedure and phony neural system. Globe Acad. Sci. Eng. Technol. Int. J. Biomed. Biol. Eng 7, 5 (2013).

10. Fan Li, Manhua Liu, and Alzheimer's Disease Infection Neuroimaging Initiative. 2018. Alzheimer's disease ailment summation influenced by various bunch thick systems that are convolutional. Automated hospital Imaging and Graphics 70 (2018), 101-110.

11. juergen Dukart, Karsten Mueller, Henryk Barthel, Arno Villringer, Osama Sabri, Matthias Leopold Schroeter, and Alzheimer's Disease Infection Neuroimaging Effort. 2013. Meta-investigation created SVM group allows precise recognition of Alzheimer's sickness across different clinical centers utilizing FDG-PET and MRI. Psychiatry Investigation: Neuroimaging 212, 3 (2013), 230-236

12. Ignacio Álvarez, Míriam López, Juan Manuel Górriz, Javier Ramírez, Diego Salas-Gonzalez, Carlos García Puntonet, and Fermín Segovia. 2008. Programmed order framework for all the bottom line of Alzheimer disorder using Component-Based SVM accumulations. In Worldwide Summit on Neural Information Operating. Springer, 402-409.

13. F Segovia, JM Górriz, J Ramírez, D Salas-González, I Álvarez, M López, R Chaves, and P Padilla. by using a GMM-based multi-variate approach. Neuroscience Letters 474, 1 (2010), 58-62.

14. Rupali S Kamathe and Kalyani Roentgen Joshi. 2018. An epic strategy influenced by free phase exam for cerebrum MR photo muscle group into CSF, WM and GM for decay identification in Alzheimer's disease sickness. Biomedical Signal control and regulation 40 (2018), 41-48

15. Seyed Hani Hojjati, Ata Ebrahimzadeh, Ali Khazaee, Abbas Babajani-Feremi, and Alzheimer's Disease Neuroimaging Initiative. 2017. Anticipating differ from MCI to advertisement resting-state that is utilizing, diagram hypothetical methodology and SVM. Diary of neuroscience method 282 (2017), 69-80.

16. Jinhua Sheng, Bocheng Wang, Qiao Zhang, Qingqiang Liu, Yangjie Ma, Weixiang Liu, Meiling Shao, and Container Chen. 2019. an impressive combined HCPMMP strategy for subsequently grouping Alzheimerâăźs and stage that is diverse customers. Public cerebrum appearance into (2019).
17. Lilia Mesrob, Benoit Magnin, Olivier Colliot, Marie Sarazin, Valérie Hahn-Barma, Bruno Dubois, Patrick Gallinari, Stéphane Lehéricy, Serge Kinkingnéhun, and Habib Benali. 2008. Recognizable proof of decay design in Alzheimer's disease issues dependent on SVM highlight determination and parcellation that is anatomical. In Global Working Area on Medical Imaging and Virtual Real Life. Springer, 124-132.

18. Benoît Magnin, Lilia Mesrob, Serge Kinkingnéhun, Mélanie Pélégrini-Issac, Olivier Colliot, Marie Sarazin, Bruno Dubois, Stéphane Lehéricy, and Habib Benali. 2009. Bolster vector order that is machine-based of's vomiting from entire cerebrum anatomical MRI. Neuroradiology 51, 2 (2009), 73-83.

19. Emilie Gerardin, Gaël Chételat, Marie Chupin, Rémi Cuingnet, Béatrice Desgranges, Ho-Sung Kim, Marc Niethammer, Bruno Dubois, Stéphane Lehéricy, Line Garnero, et al. 2009. Multidimensional order of hippocampal profile shows segregates Alzheimer's disease mellow and sickness

20. intellectual debilitation from normal maturing. Neuroimage 47, 4 (2009), 1476-1486.

21. R Chaves, J Ramírez, JM Górriz, M López, D Salas-Gonzalez, We Alvarez, and F Segovia. 2009. SVM-based Computer helped locating in the Alzheimer's condition t-test that is utilizing put possibility with identify relationship weighting. Neuroscience emails 461, 3 (2009), 293-297.

22. Claudia Plant, Stefan J Teipel, Annahita Oswald, Christian Böhm, Thomas Meindl, Janaina Mourao-Miranda, Arun W Bokde, Harald Hampel, and Michael Ewers. 2010. Mechanized identification of cerebrum decay styles dependent on MRI when it comes to hope of Alzheimer's disease. Neuroimage 50, 1 (2010), 162-174

23. Javier Ramírez, Juan Manuel Górriz, Míriam López, Diego Salas-Gonzalez, Ignacio Álvarez, Fermín Segovia, and Carlos García Puntonet. 2008. Very Early detection associated with the alzheimer sickness joining determination that is highlight component equipments. In International Discussion on Neural Suggestions Handling. Springer, 410-417

24. F Segovia, JM Górriz, J Ramírez, D Salas-González, I Álvarez, M López, Roentgen Chaves, and P Padilla. 2010. Order of functional notice pictures employing a GMM-based multi-variate approach. Neuroscience Letters 474, 1 (2010), 58-62.

25. Maciej Plocharski, Lasse Riis Østergaard, Alzheimer's disease disorder Neuroimaging effort, et al. 2016. Removal of sulcal average area and purchase of Alzheimer's sickness making use of sulcal shows. Computer tips and tasks in biomedicine 133 (2016), 35-44.

26. Ahmed Abdulkadir, Bénédicte Mortamet, Prashanthi Vemuri, Clifford R Jack Jr, Gunnar Krueger, Stefan Klöppel, Alzheimer's disease illness Neuroimaging Initiative, et al. 2011. Influences of machines heterogeneity on the convention of SVM Alzheimer's disease infection classifier. Neuroimage 58, 3 (2011), 785-792.

27. Rémi Cuingnet, Emilie Gerardin, Jérôme Tessieras, Guillaume Auzias, Stéphane Lehéricy, Marie-Odile Habert, Marie Chupin, Habib Benali, Olivier Colliot, and Alzheimer's Disease Infection Neuroimaging Step. 2011. Programmed characterization of people with Alzheimer's issues from auxiliary MRI: a study of ten campaigns using the ADNI database. neuroimage 56, 2 (2011), 766-781.

28. Daniel Schmitter, Alexis Roche, Bénédicte Maréchal, Delphine Ribes, Ahmed Abdulkadir, Meritxell Bach-Cuadra, Alessandro Daducci, Cristina Granziera, Stefan Klöppel, Philippe Maeder, et al. 2015. An evaluation of volume-based morphometry for hope of mellow weakness that is intellectual's more, Alzheimer's disease condition. NeuroImage: medical 7 (2015), 7-17

29. Maciej Plocharski, Lasse Riis Østergaard, Alzheimer's disease infection Neuroimaging effort, et al. 2016. Removal of sulcal ordinary exterior and plan of Alzheimer's disease condition utilizing sulca shows. PC procedures and work in biomedicine 133 (2016), 35-44.

30. Sandeep Chaplot, LM Patnaik, and NR Jagannathan. 2006. Order of attractive reverberation cerebrum photos utilizing wavelets as sum to assist vector equipment and neural system. Biomedical sign planning and controls 1, 1 (2006), 86-92.

31. Diego Salas-Gonzalez, Juan Manuel Górriz, Javier Ramírez, Míriam López, Ignacio Álvarez, Fermín Segovia, and Carlos García Puntonet. 2008. PC aided finding of Alzheimer's condition using bolster vector machines and characterization trees. In Foreign Discussion on Neural Information Operating. Springer, 418-425

32. Fermín Segovia, JM Górriz, Javier Ramírez, Diego Salas-Gonzalez, and Ignacio Álvarez. 2013. Very Early perseverance of Alzheimer's illness dependent on incomplete least squares and bolster vector device. Master techniques with programs 40, 2 (2013), 677-683. 
33. Andrés Ortiz, Juan M Górriz, Javier Ramírez, Francisco Jesús Martínez-Murcia, and Alzheimerâăźs Disease Neuroimaging Effort. 2013. LVQ-SVM mainly based CAD tool placed on fundamental MRI when it comes to realization regarding the Alzheimer's disorder. Example Identification Letters 34, 14 (2013), 1725-1733

34. isabelle Guyon, Jason Weston, Stephen Barnhill, and Vladimir Vapnik. 2002. Top Quality perseverance for malignancy order utilizing bolster vector machines. AI 46, 1-3 (2002), 389-422.

35. Alessandra Retico, Paolo Bosco, Piergiorgio Cerello, Elisa Fiorina, Andrea Chincarini, and Maria Evelina Fantacci. 2015. Prescient Models considering assistance Vector Machines: Whole-Brain versus local investigations of Structural MRI when you look at the Alzheimer's illness. Journal of Neuroimaging 25, 4 (2015), 552-563.

36. Antonio R Hidalgo-Muñoz, Javier Ramírez, Juan M Górriz, and Pablo Padilla. 2014. Areas of intrigue decided by SVM wrapped strategy for Alzheimer's sickness examination from separated MRI. Wildernesses in maturing neuroscience 6 (2014), 20.

37. Subrata Kar and D Dutta Majumder. 2019. a book means of Diffusion Tensor Visualization Based Neuro Fuzzy category System for very Early Detection of Alzheimer's disease Disease. Diary of Alzheimer's malady states Preprint (2019), 1-18

38. Laila Khedher, Javier Ramírez, Juan Manuel Górriz, Abdelbasse Brahim, and IA Illán. 2015. Free section examination dependent order of Alzheimer's sickness from portioned MRI records. In International Work-Conference on the Interplay among Natural and man-made calculation

39. Ali Mazaheri, Katrien Segaert, John Olichney, Jin-Chen Yang, Yu-Qiong Niu, Kim Shapiro, and Howard Bowman. 2018. EEG motions during term getting ready MCI that is anticipate transformation Alzheimer's malady. NeuroImage: medical 17 (2018), 188-197

40. Bo Cheng, Mingxia Liu, Heung-Il Suk, Dinggang Shen, Daoqiang Zhang, and Alzheimer's Disorder Neuroimaging Effort. 2015 Multimodal complex move that is regularized for MCI change expectation. Cerebrum conduct and imaging 9, 4 (2015), 913-926

41. Bo Cheng, Mingxia Liu, Dinggang Shen, Zuoyong Li, Daoqiang Zhang, and Alzheimer's Disease Condition Neuroimaging Effort. 2017. Multi-area step learning for early bottom line of Alzheimer's disease condition. Neuroinformatics 15, 2 (2017), 115-132.

42. Marcia Hon and Naimul Mefraz Khan. 2017. In direction of Alzheimer's disease illness order through exchange training. In 2017 IEEE Global discussion on Bioinformatics and Biomedicine (BIBM). IEEE, 1166-1169

43. Ke Zhou, Wenguang He, Yonghui Xu, Gangqiang Xiong, and Jie Cai. 2018. Highlight determination and go discovering for Alzheimer's disease issues medical perseverance. Systems 8, 8 (2018), 1372.

44. Bo Cheng, Mingxia Liu, Daoqiang Zhang, Dinggang Shen, Alzheimer's infection Neuroimaging effort, et al. 2018. Vigorous multi-name action highlight reading for very early dedication of Alzheimer's disease malady. Brain imaging and behavior (2018), 1-16.

45. Wei Li, Yifei Zhao, Xi Chen, Yang Xiao, and Yuanyuan Qin. 2018. Recognizing Alzheimer's Illness on Mini Dataset: An Understanding Move Viewpoint. IEEE diary of biomedical and informatics that are wellbeing2018).

46. Mehran Ahmadlou, Hojjat Adeli, and Anahita Adeli. 2010. Unique indicative EEG markers on the Alzheimer's disorder making use of perceivability diagram. Diary of sensory sign 117, 9 (2010), 1099-1109

47. Pedro Rodrigues and João Paulo Teixeira. 2011. Counterfeit systems that are neural the segregation of alzheimer's disease problems. In Global Seminar on Business Suggestions Methods. Springer, 272-281.

48. Shih-Ting Yang, Jiann-Der Lee, Chung-Hsien Huang, Jiun-Jie Wang, Wen-Chuin Hsu, and Yau-Yau Wai. 2010. Computer supported review of Alzheimer's disease sickness using different shows with artificial neural system. In Pacific Rim Global Meeting on Synthetic Intelligence. Springer,

49. 699-705.

50. Youngsang Cho, Joon-Kyung Seong, Yong Jeong, Sung Yong Shin, Alzheimer's disease disorder Neuroimaging Initiative, et al. 2012 Singular arrangement that is subject Alzheimer's infection influenced by steady getting the hang of utilizing a spatial recurrence portrayal of cortical width info. Neuroimage 59, 3 (2012), 2217-2230.

51. María Quintana, Joan Guàrdia, Gonzalo Sánchez-Benavides, Miguel Aguilar, José Luis Molinuevo, Alfredo Robles, María Sagrario Barquero, Carmen Antúnez, Carlos Martínez-Parra, Anna Frank-García, et al. 2012. Utilizing phony neural methods in medical neuropsychology: High performance in

52. Gentle impedance that is subjective Alzheimer's disease condition. Journal of Clinical and Experimental Neuropsychology 34, 2 (2012), 195-208
53. Darya Chyzhyk, Alexandre Savio, and Manuel Graña. 2014 Developmental ELM wrapper put perseverance for Alzheimer's disease malady CAD on anatomical notice MRI. Neurocomputing 128 (2014), 73-80.

\section{AUTHORS PROFILE}

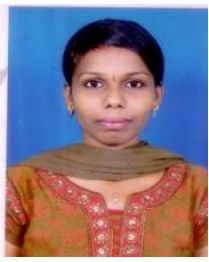

Sai Sindhuri Nasina received her B. Tech degree from Narayana Engineering College, Gudur, JNTU, Hyderabad in 2007, Masters in Computer Science and Engineering from JNTU, Anantapur in 2012 and pursuing Ph.D. in Computer Science and Engineering from Sri Venkateswara University College of Engineering, Tirupati. Her areas of interest are Big Data Analytics, Machine Learning.

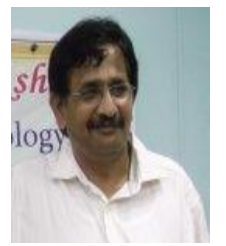

Dr. A. Rama Mohan Reddy was born in1958, received his B.Tech degree from JNT University Anantapur in 1986, Masters in Computer Science and Engineering from NIT, Warangal in 1991 and Ph.D. in Computer Science and Engineering from Sri Venkateswara University, Tirupati in 2007.He is currently working as a Professor of Computer Science and Engineering, S V University College of Engineering, Tirupati, India. His research interests are Software Engineering, Software Architecture, Cloud Computing, Operating Systems and Data Mining. 